\title{
Observation of the palm tree mode, a new MHD mode excited by type-I ELMs on JET
}

\author{
H.R. Koslowski ${ }^{1}$, B. Alper ${ }^{2}$, D.N. Borba ${ }^{3}$, T. Eich $^{4}$, S.E. Sharapov ${ }^{2}$, \\ C.P. Perez ${ }^{1}$, E. Westerhof ${ }^{5}$ and JET-EFDA contributors ${ }^{a}$ \\ ${ }^{1}$ Forschungszentrum Jülich GmbH, Association EURATOM-FZ Jülich, \\ Institut für Plasmaphysik, 52428 Jülich, Germany ${ }^{\mathrm{b}}$ \\ ${ }^{2}$ EURATOM-UKAEA Fusion Association, Culham Science Centre, Abingdon, Oxfordshire, \\ OX14 3DB, UK \\ ${ }^{3}$ Instituto Superior Técnico, Association EURATOM-IST, Av. Rovisco Pais, \\ 1049-001 Lisboa, Portugal \\ ${ }^{4}$ Max-Planck-Institut für Plasmaphysik, Association EURATOM-IPP, Boltzmannstr. 2, \\ 85748 Garching, Germany \\ ${ }^{5}$ FOM-Institute for Plasmaphysics 'Rijnhuizen', Association EURATOM-FOM, \\ PO Box 1207, 3430 BE Nieuwegein, The Netherlands ${ }^{\mathrm{b}}$
}

Received 26 July 2004, accepted for publication 31 January 2005

Published 22 February 2005

Online at stacks.iop.org/NF/45/201

\begin{abstract}
A new MHD mode has been discovered during type-I ELMy H-modes in the Joint European Torus (JET) tokamak. This mode is excited by the perturbation of the edge plasma due to the edge localized mode (ELM). It is radially and poloidally well localized and has the toroidal mode number $n=1$ and the poloidal mode number $m=3$. The mode appears in different plasma configurations and in a wide range of global plasma parameters as long as the rational $q=3$ surface is located in the ELM perturbed region. A possible explanation for the new mode is as the remnant of a magnetic island created by edge ergodization during the ELM. Consequences for the understanding of the ELM process itself are discussed.
\end{abstract}

PACS numbers: 52.30Cv, 52.35Vd, 52.55Tn

(Some figures in this article are in colour only in the electronic version)

\section{Introduction}

An operating regime of particular interest for the tokamak is the H-mode [1] where the energy confinement is about doubled with respect to normal operating conditions, observed in tokamaks equipped with a poloidal divertor. The increased energy content of $\mathrm{H}$-mode plasmas is stored in the so-called pedestal, causing the profile gradients at the plasma edge of these plasmas to become rather large and leading to the destabilization of edge localized modes (ELMs) [2]. ELMs show up as periodic bursts of magnetic perturbations which enhance the transport in the gradient zone at the edge and lead to losses of plasma energy of up to several per cent of the stored energy content. The high transient heat loads associated with the ELMs may cause strong erosion of the divertor target plates

a See annex 1 of Paméla J. et al 2003 Overview of recent JET results, OV-1/1.4 Fusion Energy 2002: Proc. 19th Int. Conf. (Lyon, 2002) (Vienna: IAEA) Nucl. Fusion 431540.

b Member in the Trilateral Euregio Cluster, TEC. and could considerably shorten the lifetime of plasma facing components [3].

Various theories have been put forward in order to explain ELMs [4,5]. A quite successful approach is the description of the ELM in terms of a coupled ballooning and peeling instability [6]. This model for the ELM is based on linear MHD stability theory, i.e. it describes the onset of the ELM, but does not describe the mechanism of the explosive increase of transport during the short ELM bursts $\left(\tau_{\mathrm{ELM}} \approx 200 \mu \mathrm{s}\right)$.

One of the main topics in present tokamak research, where the physical basis for operation of the next step fusion device, ITER [7], has to be explored, is to find means to control ELMs and operate the plasma in a state where on one hand the energy confinement is sufficient to allow the extrapolation to a burning fusion plasma, while on the other hand the power loss per ELM is tolerable for the divertor [8].

During plasma operation at the Joint European Torus (JET) tokamak a new MHD mode has been observed. This mode is excited by type-I ELMs and can probably give new 
Table 1. Summary of the discharges discussed in this paper.

\begin{tabular}{|c|c|c|c|c|c|c|}
\hline Pulse & $B_{\mathrm{t}}$ & $I_{\mathrm{p}}$ & $q_{95}$ & $\delta_{\mathrm{u}}$ & $\delta_{1}$ & Description \\
\hline 52011 & 2.7 & 2.5 & 2.9 & 0.44 & 0.35 & $\begin{array}{l}\text { ITER-like high } \\
\text { triangularity }\end{array}$ \\
\hline 52148 & 2.4 & 2.5 & 3.0 & 0.19 & 0.24 & $\begin{array}{l}\text { RI-mode with } \mathrm{Ar} \\
\text { seeding }\end{array}$ \\
\hline 58982 & 2.5 & 3.0 & 2.8 & 0.19 & 0.25 & $\begin{array}{l}\text { Standard ELMy } \\
\text { H-mode }\end{array}$ \\
\hline
\end{tabular}

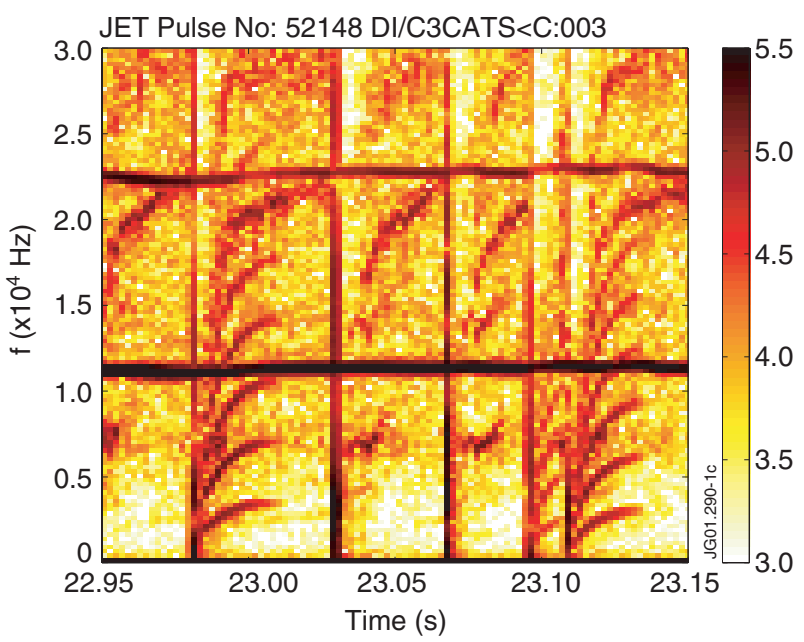

Figure 1. Typical signature of palm tree modes as seen in a magnetic spectrogram. The vertical lines correspond to the broadband magnetic perturbation of ELMs, after three of them a palm tree mode appears.

insight in the ELM process itself. In the following the experimental observations are described. Some underlying physics which might be connected to the understanding of the new mode is also discussed.

\section{Experimental observations}

Palm tree modes have been observed in a wide range of JET plasma parameters and for various plasma configurations, as long as type-I ELMs were present. Table 1 gives an overview on the plasma configurations of the discharges showing palm tree modes which are discussed in this paper.

\subsection{Frequency and time behaviour}

Figure 1 shows a spectrogram calculated from the fast sampled data of a magnetic pick-up coil situated near the plasma. The horizontal lines at frequencies of 11 and $22 \mathrm{kHz}$ are due to MHD activity in the plasma core, the sawtooth precursor mode and its first harmonic at twice the frequency. The five vertical structures are due to the short-lasting broadband magnetic perturbation of the type-I ELMs. Modes which start immediately after the ELM and increase in frequency are visible after three of the ELMs. This typical signature of this mode, which resembles the leaves of a palm tree, inspired the naming.

The modes show many harmonic frequencies (up to six harmonics can be seen in the figure), the spectrogram resembles the so-called snakes, observed at the $q=1$ [9] and
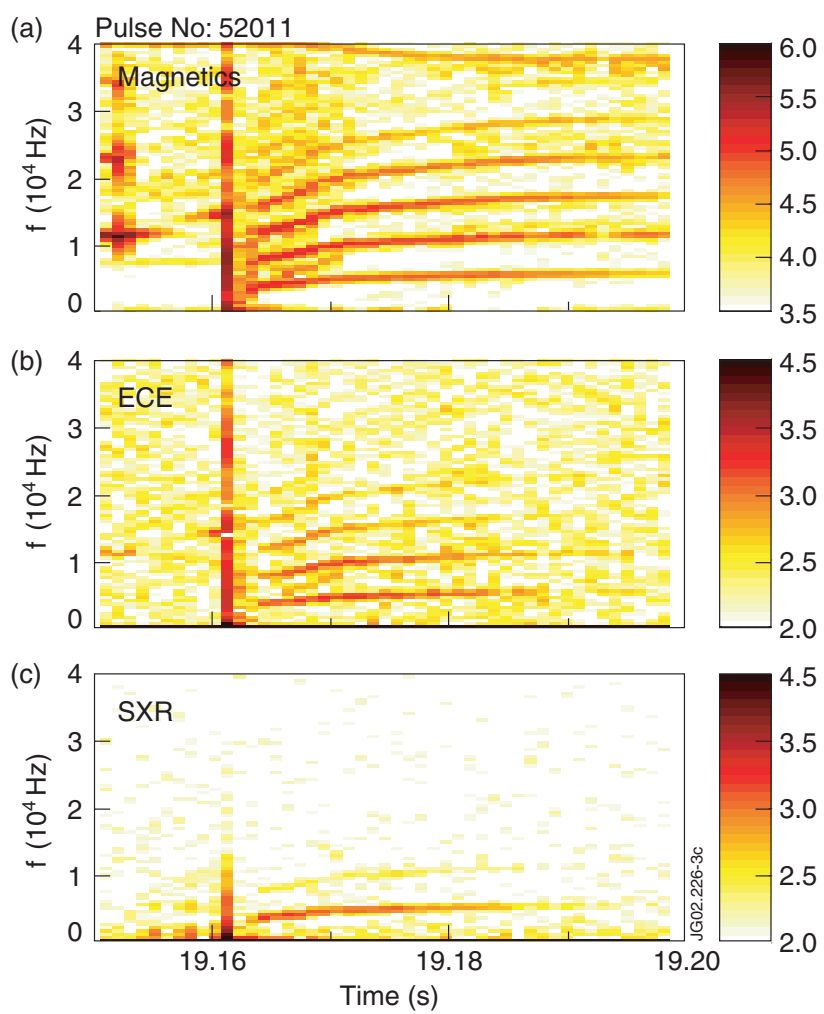

Figure 2. Spectrograms of signals from various diagnostics showing the palm tree mode. (a) magnetic pick-up coil, (b) ECE emission, (c) SXR emission.

$q=2[10]$ surfaces. The modes last for 5-50 ms and decay afterwards. An ELM event occurring during a palm tree mode stops it immediately (see palm tree mode after fourth ELM in figure 1, which is stopped by the fifth ELM).

The mode has been detected not only with magnetic pick-up coils. Electron cyclotron emission (ECE) electron temperature measurements as well as the detection of the soft X-ray (SXR) emission from the plasma detect that mode, too. This is illustrated in figure 2 where spectrograms of fast sampled $\left(f_{\text {samp }}=250 \mathrm{kHz}\right)$ signals of $(a)$ a magnetic pick-up coil mounted at the low-field side of the torus, $(b)$ the ECE emission originating at a radial position of $R=3.84 \mathrm{~m}$, and (c) the SXR emission measured along a line of sight tangential near the edge are shown. Figure 3 shows the poloidal locations and lines of sight of the various diagnostics.

The frequency of the mode increases in time. At start, i.e. immediately after the ELM, the frequency can be close to zero or have some finite value.

A third example of a palm tree mode is shown in figure 4. Here the magnetic spectrogram is plotted in the upper part of the figure. The corresponding $\mathrm{D}_{\alpha}$ emission measured in the divertor is shown in the lower part. The ELMs in this example have a double structure (compound ELMs). Both palm tree modes are triggered by the second ELM which has a weaker magnetic perturbation as can be seen from the lack of the broadband structure at the ELM crash in the spectrogram (vertical lines). Note that the second palm tree mode is not triggered by the sawtooth crash (the line at $13 \mathrm{kHz}$ is the sawtooth precursor mode, which stops at the crash), but from an ELM occurring $1 \mathrm{~ms}$ later when the sawtooth heat pulse 


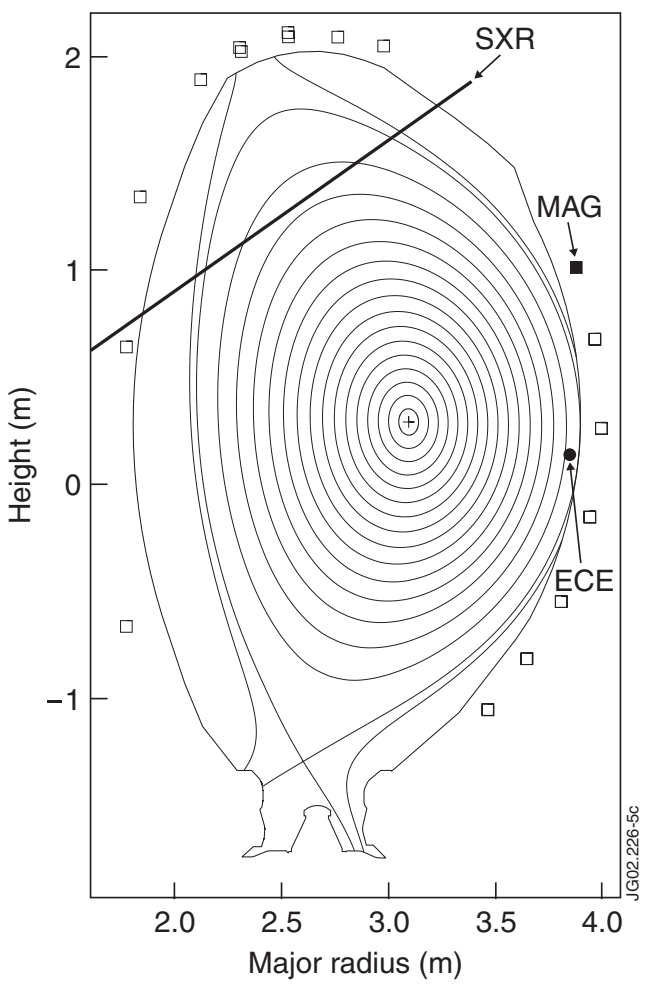

Figure 3. Location of the magnetic pick-up coil, the volume where the detected ECE emission emerges, and the line of sight of the SXR camera used in figure 2 .

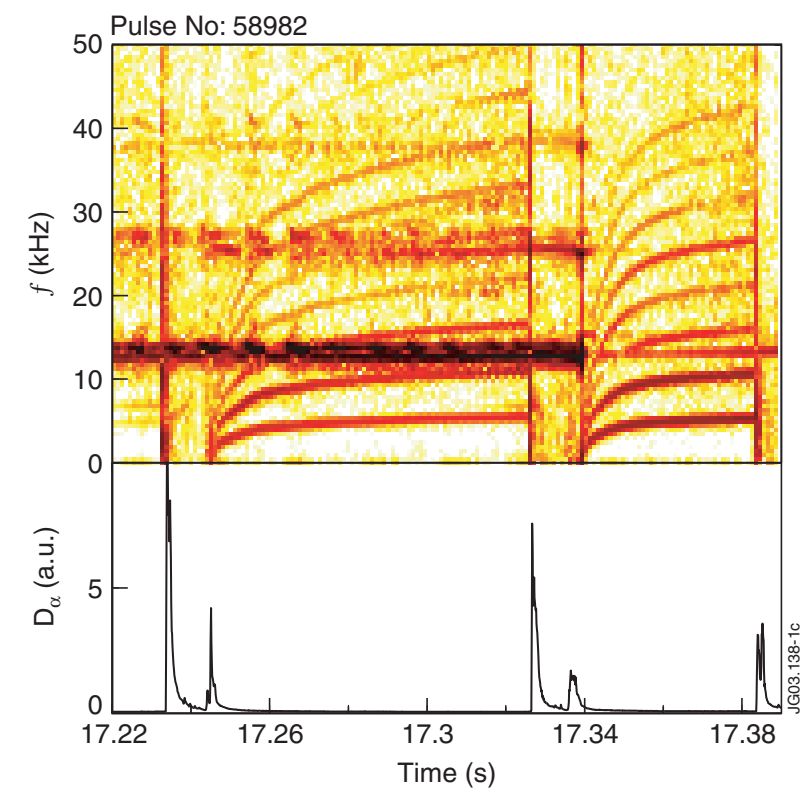

Figure 4. Another example of palm tree modes in a type-I ELMy H-mode plasma. During the second palm tree mode only very little other MHD activity is present.

reaches the edge. Both mode oscillations are suddenly stopped when another ELM occurs.

The absence of strong core MHD activity during the second palm tree mode between 17.34 and 17.38 s (only a weak sawtooth precursor is present at that time) allows the Fourier spectrum to be plotted (see figure 5), where the multiple harmonics of the palm tree mode are clearly visible.

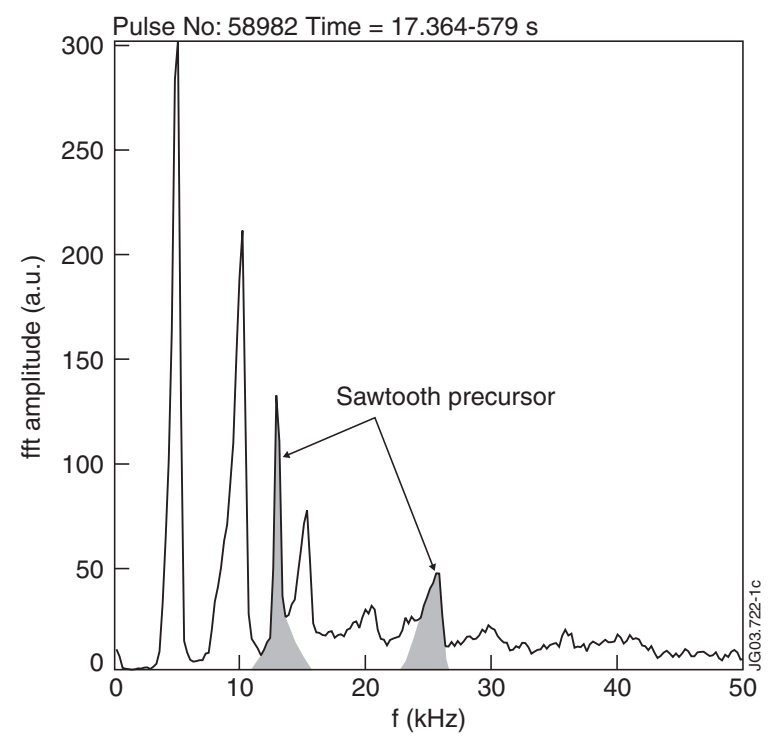

Figure 5. Amplitude spectrum of the same discharge as figure 4, averaged between $t=57.364$ and $57.379 \mathrm{~s}$. The multiple harmonics of the palm tree mode at $5,10,15,20,25,30$ and $35 \mathrm{kHz}$ are clearly visible. The sawtooth precursor at $13 \mathrm{kHz}$ and its first harmonic at $26 \mathrm{kHz}$ cause only a minor distortion of the signal.

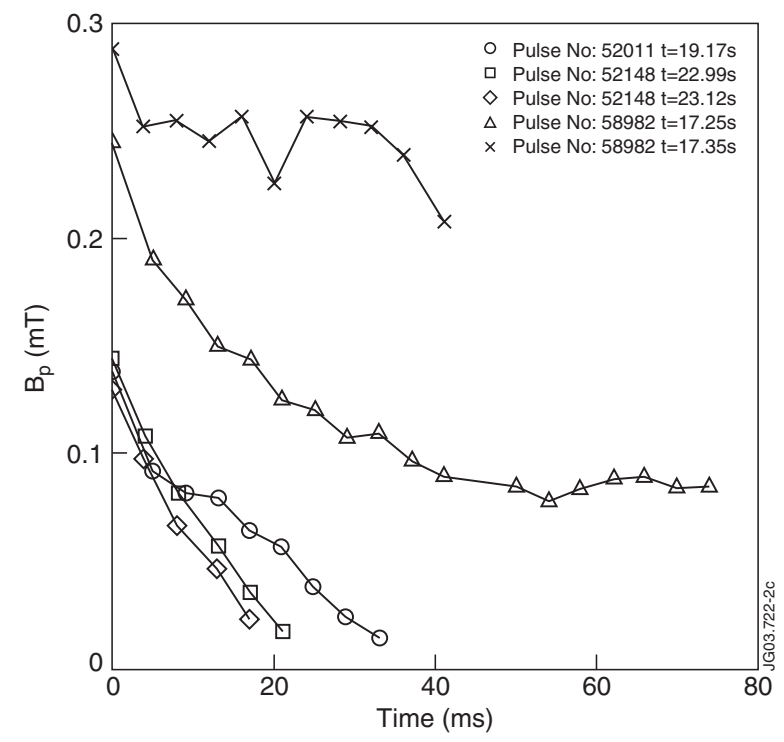

Figure 6. Magnetic perturbation amplitudes of all palm tree modes shown in this paper. The amplitudes are measured at the pick-up coil location.

The calibrated amplitudes of the magnetic perturbations of the various palm tree modes shown in figures 1,2 and 4 are plotted in figure 6 . The amplitudes are corrected for frequency and determined at the location of the pick-up coil. The figure shows that the mode amplitude decays in time, i.e. there is a mechanism which stabilizes the palm tree modes. It can be seen that the modes in discharge \#58982 (figure 4) are stopped at a quite large amplitude by the next ELM event.

\subsection{Mode numbers}

The mode numbers of the palm tree mode have been determined by phase comparison of the fluctuations measured 


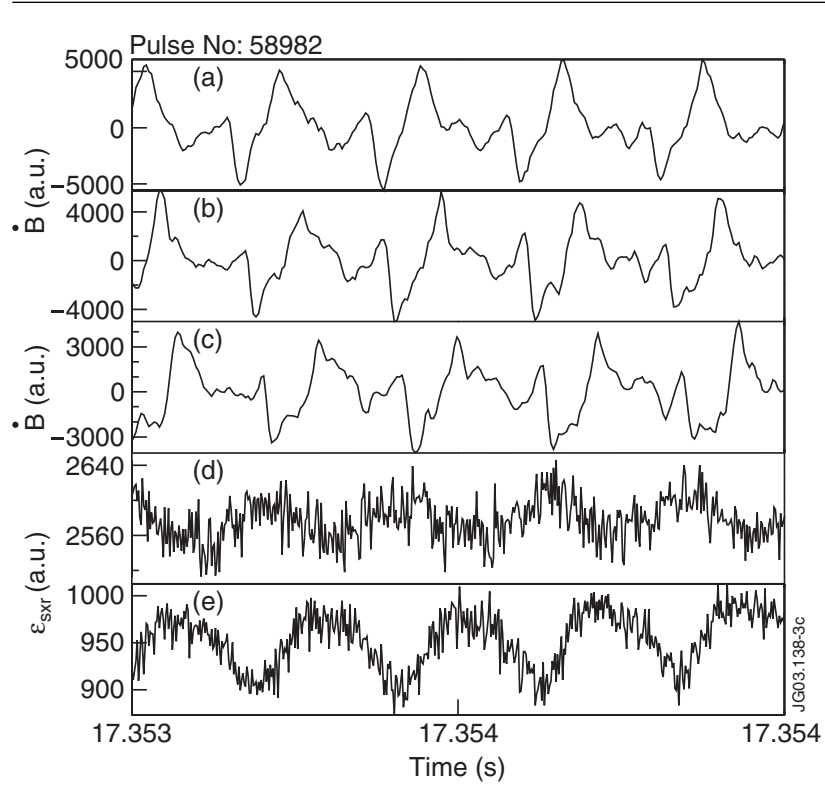

Figure 7. Signals from three poloidal pick-up coils situated in the outboard limiter at the same toroidal angle and separated poloidally by $20^{\circ}(a)-(c)$, and two SXR signals $(d)$ and $(e)$ from an horizontal fan viewing at the same toroidal position.

by toroidal and poloidal arrays of magnetic pick-up coils using the CATS diagnostic on JET $[11,12]$. The toroidal mode number of the lowest frequency component of the palm tree mode has been identified to be $n=1$. The corresponding poloidal mode number is $m=3$. Therefore, the palm tree mode is located at the rational $q=3$ surface. The harmonic frequencies visible in figures 1 and 4 have toroidal mode numbers $n=2,3,4, \ldots$ and the poloidal mode number of the second harmonic is $m=6$, the poloidal mode numbers of higher harmonics could not be determined due to the limited angular resolution of the used coil array. The large number of harmonic frequencies show that the signal modulation due to this mode is strongly non-sinusoidal.

\subsection{Mode structure and propagation}

Figure 7 shows the signals from a set of three fast pick-up coils situated in one poloidal cross section in the outboard limiter and two channels of a SXR camera located at the same toroidal position. The viewing lines of the SXR camera intersect the plasma below the pick-up coils. The signals of the pick up coils show that the structures visible on them proceed in time from top to bottom $(a)-(c)$. This means that at a fixed toroidal location the mode propagates poloidally downward, i.e. in direction of the ion diamagnetic drift.

The SXR signals of two neighbouring channels $(d)$ and $(e)$ have a phase inversion in-between. Therefore, the palm tree mode has a tearing mode structure, i.e. it is a magnetic island which requires reconnection. The O-point of the island is where both SXR signals come closest together, i.e. at the minima in the upper SXR signal.

\subsection{Localization of the mode}

In figure 8, four neighbouring channels of the ECE temperature diagnostic are plotted. The channels cover a part of the edge
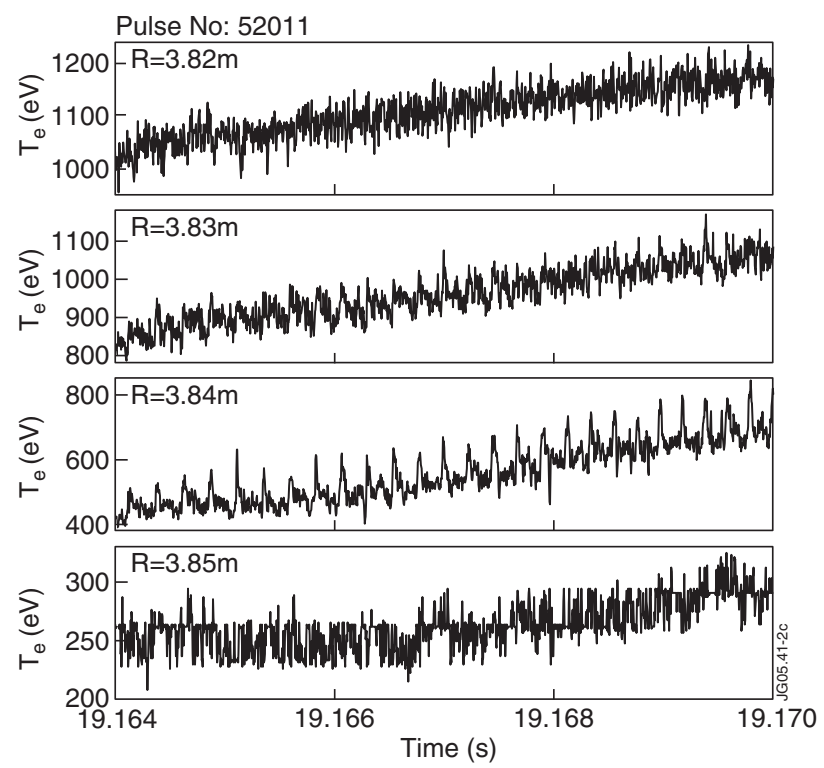

Figure 8. Four ECE channels measuring the electron temperature in the pedestal region.

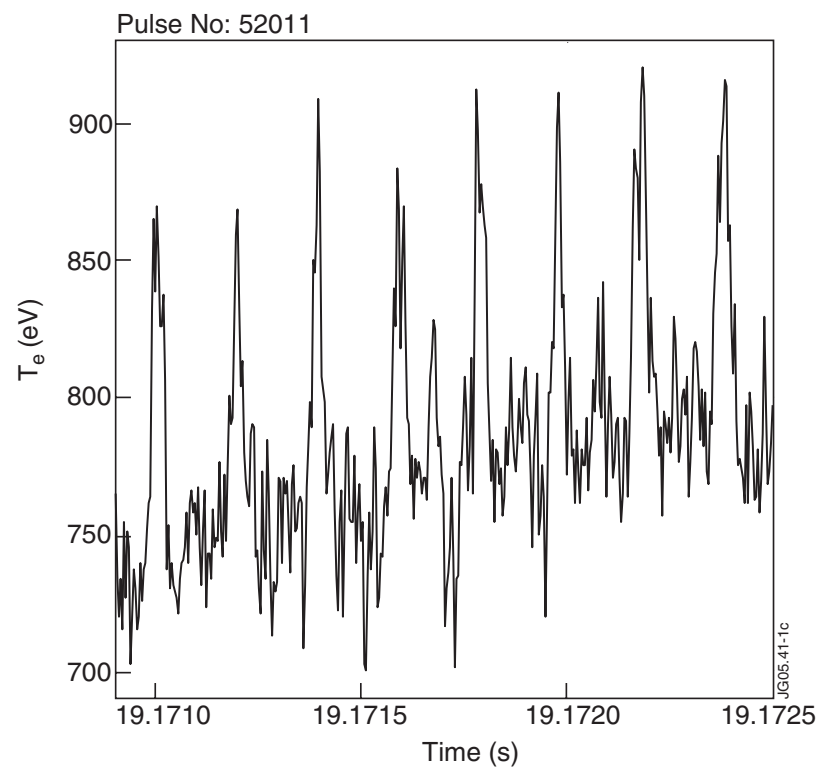

Figure 9. Enlarged view of the ECE channel at $R=3.84 \mathrm{~m}$ shown in figure 8.

pedestal region from $R=3.82$ to $3.85 \mathrm{~m}$ with a spatial separation of $1 \mathrm{~cm}$. The strongest modulation is visible on the time trace measured at $R=3.84 \mathrm{~m}$, the one at $R=3.83 \mathrm{~m}$ shows a much smaller modulation. Both other channels do not see the mode. Therefore, the radial extension of the palm tree mode is $w_{\mathrm{r}} \approx 1-2 \mathrm{~cm}$.

The mode is not only radially well localized, but is poloidally localized as well. Figure 9 shows an enlarged view of a part of the time trace measured at $R=3.84 \mathrm{~m}$. As can be concluded from the high number of harmonics in the spectrum, the modulation of the time trace is non-sinusoidal. The time trace of the electron temperature shows narrow spikes with a higher electron temperature than outside the spike 


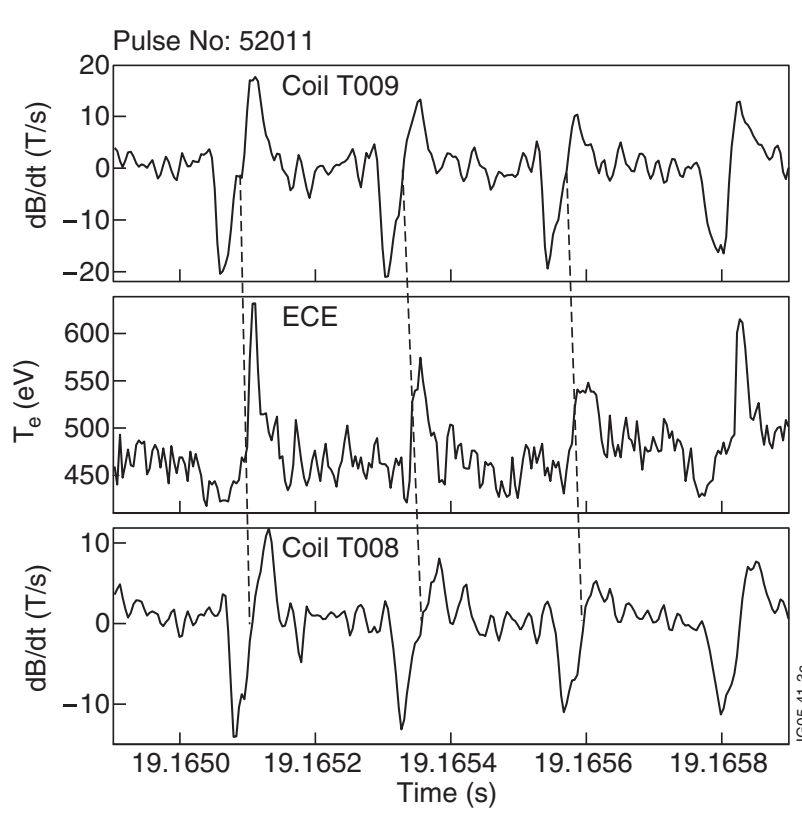

Figure 10. Comparison of an ECE channel measuring at $R=3.84 \mathrm{~m}$ with the signals of two pick-up coils which are toroidally displaced to both sides.

structures. The width of the spikes amounts to about 10-20\% of the modulation period. The maximum value of the electron temperature within the spikes is lower than the temperature has been before the preceding ELM collapse which triggered the palm tree mode. The $q=3$ surface in this discharge has a poloidal circumference of $7 \mathrm{~m}$ and the poloidal projection of the palm tree mode has (because $m=3$ ) three island structures. From the width of the structure one can estimate a poloidal extent of $w_{\Theta} \approx 23-46 \mathrm{~cm}$ for each structure.

\subsection{Further observations on the mode structure}

In figure 10, two signals from magnetic pick-up coils are plotted together with the ECE signal showing the hot spikes (figure 9). During the time interval shown, no background MHD activity was present in the plasma, as can be seen in the spectrogram (figure 2). The pick-up coils are mounted at the inboard side of the vacuum vessel at $z=1 \mathrm{~m}$ and toroidally separated by $33^{\circ}$. The ECE diagnostic is mounted in the same octant between both coils. The volume observed by the ECE diagnostic is located below the pick-up coils at $z \approx 0.2 \mathrm{~m}$. Both magnetic signals $((a)$ and $(c))$ are very similar and show the same features.

The polarity of the coil signals is such, that a current flowing in direction of the plasma current (i.e. counterclockwise when viewed from top of the torus) induces a positive voltage in the coils. The negative and positive spikes on the magnetic signals can therefore be attributed to the magnetic perturbation of the palm tree mode. As has already been shown in figure 7 that the palm tree mode has an island structure, i.e. it is a tearing mode. In order to create such a magnetic topology one needs to drive a positive current in the $\mathrm{X}$-point and/or a negative current in the O-point. Because the pick-up coils detect $\mathrm{d} B_{\Theta} / \mathrm{d} t$, the negative spike in the signals implies that the poloidal magnetic field at the coil decreases in time. A negative signal is therefore equivalent to a negative

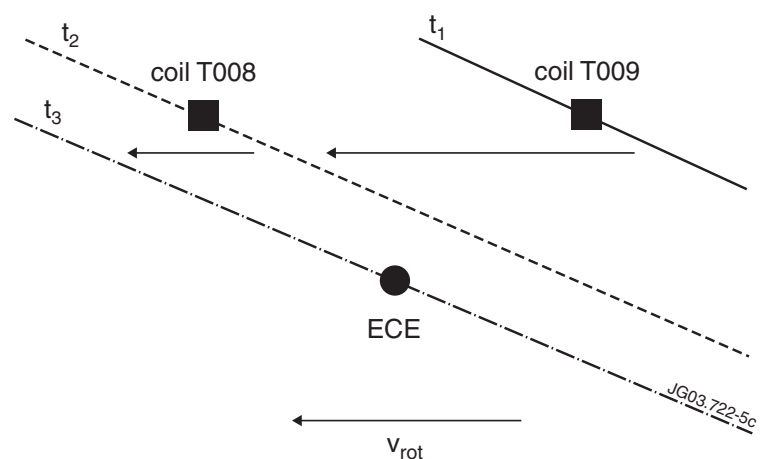

Figure 11. Schematic drawing of the positions of the pick-up coils and the ECE channel. The diagonal lines represent a field line at three times $t_{1}<t_{2}<t_{3}$.

current which approaches the coil. This current has reached its closest distance to the coil when the signal is zero again. At that time the O-point of the island has its closest distance to the pick-up coil. The dashed lines in figure 11 connect those points of both signals.

The lower magnetic signal (c) has a time delay of about $15 \mu \mathrm{s}$, which is the time required to move one field line from the first to the second coil position, toroidally separated by $33^{\circ}$ in direction of the plasma current. The mode rotation in toroidal direction is in qualitative agreement with the outermost CX rotation measurement at $R=3.81 \mathrm{~m}$. The positive (hot) spike in the ECE signal $(b)$ appears even later than the O-point signature on the second coil signal $(c)$. This is explained due to the different vertical positions of the pick-up coils and the volume measured by the ECE diagnostic. A schematic drawing of the geometry is shown in figure 11 . Here the various positions are shown together with a magnetic field line at various times $t_{1}<t_{2}<t_{3}$. At $t_{1}$ the structure (e.g. the O-point) is positioned closest to the first coil T009. Because the field line has only a small inclination (note that $q$ is 3), it reaches first the second coil T008 at $t_{2}$ before it crosses the ECE volume at time $t_{3}$. The figure assumes only toroidal rotation, but poloidal rotation due to drifts cannot be excluded in the pedestal region. Anyhow, this will not change the above argumentation which also holds in case the plasma rotates only in poloidal direction. Therefore one can conclude, that the hot spikes in the ECE signal are located at the O-points, i.e. the islands have a higher temperature then the outside regions.

It is important to note that the maximum temperature of the spikes is still lower than the temperature at the same radial position has been before the ELM crash.

A further detail of the mode structure can be concluded from the narrow width of the hot spikes compared to the poloidal separation of two adjacent O-points, which amounts to more than $2 \mathrm{~m}$. This suggests, that the (relatively small) islands are connected via Y-points and a neutral line, rather than via $\mathrm{X}$-points.

\subsection{Dependence on global plasma parameters}

The first observation of a palm tree mode was in an impurity seeded discharge [13], where the radiation from the injected medium $Z$ atoms reduces the divertor load. An inspection of a 

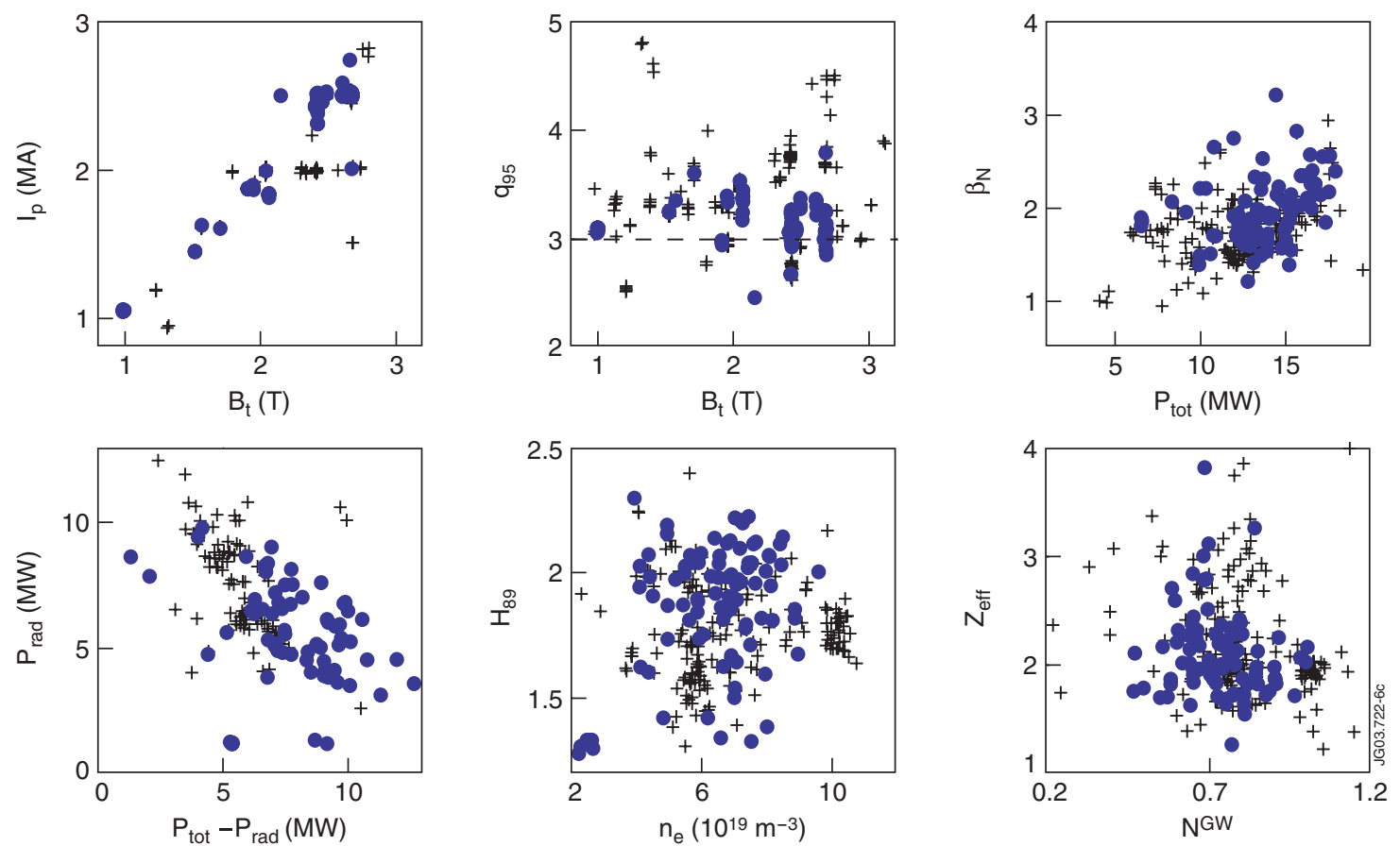

Figure 12. Overview on various global discharge parameters of type-I ELMy H-modes where palm tree were observed $(\bullet)$ and discharges without palm tree modes (+).

large number of ELMy H-modes with type-I ELMs revealed, that the new mode appears in various plasma configurations. It is observed in standard H-modes, impurity seeded plasmas, pellet fuelled plasmas, and ITER-like shaped plasmas with high triangularity.

The global plasma parameters where palm tree modes occurred are summarized in figure 12. The full circles represent time windows with palm tree modes, the crosses are without. The mode appears for different combinations of plasma current and toroidal magnetic field, as long as the $q=3$ surface is located near the plasma edge (see $q_{95}$ graph in the figure). The total heating power can vary a lot, as long as the power is above the threshold to produce type-I ELMs. Palm tree modes seem to be more likely to occur at higher input powers and higher confinement quality factors $\left(H_{89}\right)$. Finally, figure 12 shows that the effective charge and the electron density normalized by the Greenwald density could vary by large amounts over the domain for which palm tree modes are observed. Figure 12 also shows that palm tree modes only occur sometimes, i.e. there is no unambiguous parameter among the ones shown in the figure which clearly predicts the onset of palm tree modes. The observations imply that there is still some randomness for the new mode to occur.

\section{Discussion}

The observed features of the palm tree mode can be summarized as follows:

- The palm tree mode occurs in type-I ELMy H-mode plasmas.

- It is triggered by an ELM crash.
- The rational $q=3$ surface has to be located close to the plasma edge where the ELM perturbation is strong.

- An ELM collapse occurring during a palm tree mode will stop it immediately.

- The mode frequency increases in time and saturates. Sometimes the frequency even starts at zero.

- The amplitude of the modes decreases in time.

- It has snake-like signatures, i.e. the spectrum consists of many harmonic frequencies.

- The mode numbers are $n=1,2,3, \ldots$ and $m=$ $3,6,9, \ldots$

- The mode propagates in the ion diamagnetic drift direction.

- The mode is radially localized within $\Delta R \approx 1-2 \mathrm{~cm}$. Its poloidal extension is small compared to the circumference of the $q=3$ surface.

- The palm tree mode has a tearing mode structure, i.e. it requires reconnection to be created.

- It is a hot island, the temperature at the O-point is higher than outside.

The palm tree mode shows some similarity to the so-called snakes, which were observed in JET at the $q=1$ [9] and $q=2$ [10] surfaces. The observation of a snake-like mode at the $q=3$ surface has been reported recently in [14], but this mode (the so-called picket fence mode) was observed in optimized shear scenarios and found to disappear after the $\mathrm{L}-\mathrm{H}$ transition, whereas the mode reported here does only occur in H-mode plasmas. Another difference between the palm tree mode and the picket fence mode is the different directions of rotation of both modes. As can be seen from figures 10 and 11, the palm tree mode propagates in direction of the plasma current, i.e. it rotates with the bulk plasma driven by the neutral beam injection. On the contrary, the picket 


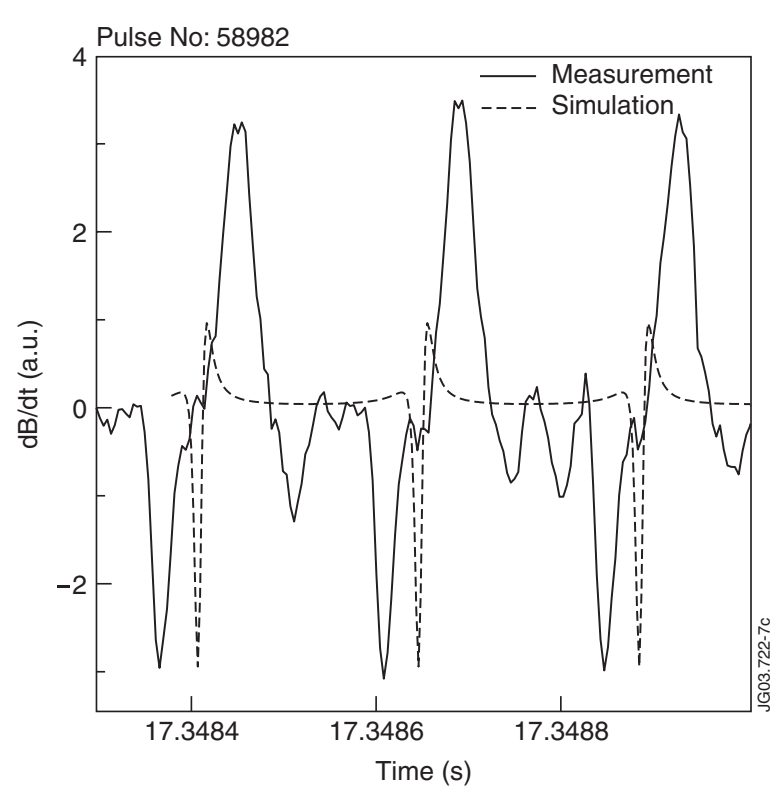

Figure 13. Comparison of the signal from a poloidal pick-up coil $(-)$ with the simulated signal resulting from the current filament of a $q=3$ snake (- - - ). The smaller structures in the measured signal are caused by a weak internal kink mode rotating with $12 \mathrm{kHz}$ (see spectrogram in figure 4 between 17.34 and $17.38 \mathrm{~s}$ and FFT amplitude in figure 5 .

fence mode is reported to rotate opposite to the plasma bulk rotation [14].

The magnetic signatures of the picket fence mode are somewhat different to the ones of the palm tree mode. The picket fence mode could be modelled quite well by helical current filaments. The comparison of the magnetic signals of such a current filament with the signal from a palm tree mode is shown in figure 13. The agreement is not very good. The palm tree mode (full curve) has a much wider structure than the perturbation from a current filament (dashed line). The current perturbation causing the palm tree mode has therefore to be more extended than the one of the picket fence mode.

The frequency evolution of the mode suggests that the ELM not only leads to loss of particles and energy, but momentum is lost as well. The rotation of the palm tree mode after the ELM is stopped, or at least considerably slowed down. The mode frequency seen by a pick-up coil is $\omega_{\text {mode }}=n \omega_{\phi} \pm m \omega_{\theta}+\omega_{e}^{*}$, where $\omega_{\phi, \theta}$ are the toroidal and poloidal plasma rotation frequencies, and $\omega_{e}^{*}$ is the diamagnetic drift frequency [15]. An estimate of $\omega_{e}^{*} / 2 \pi$ gives $3.5 \mathrm{kHz}$ for high density $\mathrm{H}$-modes, where lots of the palm tree mode observations were made. Even a change by $50 \%$ due to the collapse of the edge pressure gradient (what is quite normal for JET type-I H-modes) could not explain the frequently observed start of the palm tree mode at zero frequency without a considerably change in toroidal plasma rotation. This finding is in good agreement with spectroscopic measurements on the COMPASS tokamak, where a change in toroidal rotation velocity after the ELM crash has been reported [16].

In order to understand the phenomenon of the palm tree modes one has to find a mechanism which is triggered or initiated by the perturbation of the plasma edge due to an ELM collapse. One likely explanation might work as follows.
The magnetic perturbation associated with the ELM collapse ergodizes the magnetic field lines at the plasma edge. It is known from Hamiltonian theory, that situations exist where islands (i.e. regions where regular flux surfaces exist) are embedded in ergodic regions [17]. Depending on the degree of ergodization, the islands vary in size and at larger levels of magnetic perturbation no residual island structures exist at all.

A similar situation was faced recently when the anticipated field structure of the dynamic ergodic divertor (DED) on the TEXTOR tokamak [18], a special device which is aimed to generate an ergodic field in the plasma, has been modelled. The results showed, that depending on the amplitude of the perturbation field situations without full ergodization, where island structures within ergodic regions exist, can easily be produced [19].

From the experimental observations it is clear that the ELM crash is the trigger of the palm tree modes. The mechanism itself, which creates the island cannot directly be inferred from the measurements, because the timescale is rather short and the ELM crash perturbs the signals. Assuming that the idea of ergodization is right, the palm tree mode can be explained to be the remnant of a $(3,1)$-island generated by incomplete edge ergodization due to the magnetic perturbation of the ELM.

The hot island structures observed experimentally result from an improved confinement within magnetic islands, as has been previously measured for large $(2,1)$ tearing modes [20]. The islands are not necessarily regions where the profiles are completely flat. This improved confinement inside the island explains the observed higher temperature in the spikes (i.e. O-points), which results because the heat from the ergodized region outside the island structures is lost much faster during the ELM crash.

Previous work on JET showed that the local electron temperature within a large $m / n=2 / 1$ island before a disruption was flattened [21]. That finding is not necessarily a contradiction to the present observation of hot island structures. The timescale of formation of the palm tree mode (during the ELM crash, $\approx 100 \mu \mathrm{s}$ ) is much shorter than the timescale for the growth of the $2 / 1$ tearing mode. The higher temperature of the palm tree islands results from the faster heat removal from the surrounding area, assisted by a better confinement due to intact flux surfaces inside the island. As can be seen from figure 2 , the temperature perturbation equilibrates faster (transport timescale) than the decay of the magnetic perturbation (resistive timescale) of the mode.

Furthermore, the self stabilization of the palm tree modes can be explained by a higher Ohmic current flowing in the O-point of the mode (due to the better conductivity), thus compensating for the loss of current within an islands O-point. A further contribution to the stabilization may be come from a negative tearing parameter $\Delta^{\prime}$ of the induced island.

When edge ergodization is the key to understand the palm tree modes, the question arises whether these modes with higher poloidal mode numbers do exist as well, provided that the, e.g. $q=4$ surface is located in the ELM perturbed region. Indeed, two examples of palm tree modes which are located most likely at the $q=4$ surface have been found. Mode analysis using the CATS diagnostic on JET results in $m=4$ and $n=1$, although the the determination of the poloidal mode 
number is somewhat uncertain. Anyhow, the main difference of the few discharges with $q=4$ palm tree modes when compared to the discharges discussed here is the value of $q_{95}$, which is indeed in the range $4-4.2$, whereas the $q=3$ observations have a $q_{95}$ close to 3 (see table 1 ).

The fact that palm tree modes are not always present after type-I ELMs shows that the degree of edge ergodization varies, not only between discharges, but even within one discharge from one ELM to the next one (see e.g. figure 1). Indeed, in many cases some kind of noise is visible in magnetic spectrograms directly after the ELM. When one performs mode number analysis in these cases, the mode number spectra reveal that the noisy structures split up into the various $m$ and $n$ values, nicely ordered from low to high, as has been observed for the palm tree modes. This may be an indication, that similar structures are generated by the ELM, but the islands do not persist, probably due to a higher level of edge ergodization.

The question arises, whether the varying degree of edge ergodization is related to the transport during the ELM. Unfortunately, no absolute ELM amplitude can be derived because the signals from the pick-up coils saturate. Some attempts to correlate the ELM energy loss with the appearance of palm tree modes were done, but first results did not show any significant difference in the energy loss of ELMs with and without palm tree modes following the ELM crash. Furthermore, there is no noticeable change in the global energy confinement found, comparing ELMs without and followed by a palm tree mode.

\section{Summary and conclusion}

The palm tree mode can be regarded as an ELM postcursor mode. It is generated due to the magnetic perturbation of the ELM and occurs in a wide variety of plasma conditions, as long as the rational $q=3$ surface is located in the ELM perturbed region. There is strong evidence that the palm tree mode results from edge ergodization, as otherwise the hot islands would be difficult to explain. This favours, in particular, models of the ELM collapse which are based on the assumption of field line ergodization [22].

The scaling of the type-I ELM energy losses to ITER [23] is much more favourable when parallel transport along ergodized field lines is assumed. This assumption could be supported by the observation of the new mode in case the proposed model of the palm tree mode turns out to be correct.

ELM precursor modes [24] often have higher toroidal mode numbers, but further work could aim to investigate a possible connection between both processes, or show that the excitation of palm tree modes is not related to the modes causing the ELM crash.

Up to now there have been no observations of palm tree modes reported from other tokamaks like ASDEX Upgrade or DIII-D. Future work on these machines should aim at the identification of palm tree modes, or show that they definitely do not exist. In the latter case it may be important to understand why they are only observed in JET ELMy H-mode plasmas.

\section{Acknowledgment}

This work has been conducted under the European Fusion Development Agreement.

\section{References}

[1] Wagner F. et al 1982 Phys. Rev. Lett. 491408

[2] Zohm H. 1996 Plasma Phys. Control. Fusion 38105

[3] Federici G. et al 2001 Nucl. Fusion 411967

[4] Connor J.W. 1998 Plasma Phys. Control. Fusion 40191

[5] Connor J.W. 1998 Plasma Phys. Control. Fusion 40531

[6] Suttrop W. 2000 Plasma Phys. Control. Fusion 42 A1

[7] Aymar R., Barabaschi P. and Shimomura Y. 2002 Plasma Phys. Control. Fusion 44519

[8] Saibene G. et al 2002 Plasma Phys. Control. Fusion 441769

[9] Gill R.D., Edwards A.W., Pasini D. and Weller A. 1992 Nucl. Fusion 32723

[10] Alper B., Allfrey S.J., Borba D., Conway G.D., Gill R.D., Hawkes N.C., Hender T.C., Smeulders P. and Wesson J.A. 1999 European Conference Abstracts 23J 173

[11] Blackler K. and Edwards A.W. 1994 IEEE Trans. Nucl. Sci. 41111

[12] Smeulders P., Conway G.D., Alper B., Balet B., Bartlett D.V., Borba D., Deliyanakis N., Hender T.C. and Kwon O.J. 1999 Plasma Phys. Control. Fusion 411303

[13] Dumortier P. et al 2002 Plasma Phys. Control. Fusion 441845

[14] Huysmans G.T.A. et al 1999 Nucl. Fusion 391489

[15] Klüber O., Zohm H., Bruhns H., Gernhardt J., Kallenbach A. and Zehrfeld H.P. 1991 Nucl. Fusion 31907

[16] O'Connell R., Carolan P.G., Bunting C.A., Conway N.J., Leahy P. and Todd T.N. 1997 European Conference Abstracts 21A 273

[17] Abrahams R. and Marsden J.E. 1978 Foundations of Mechanics 2nd edn (Reading, MA: The Benjamin/Cummings)

[18] Finken K.H. (ed) 1997 Dynamic ergodic divertor (15 articles) Fusion Eng. Des. 37335 (special issue)

[19] Eich T., Reiser D. and Finken K.H. 2000 Nucl. Fusion 401757

[20] de Vries P.C., Waidmann G., Krämer-Flecken A., Donné A.J.H. and Schüller F.C. 1997 Plasma Phys. Control. Fusion 39439

[21] Wesson J.A. et al 1989 Nucl. Fusion 29641

[22] Itoh S.-I., Itoh K., Zushi H. and Fukuyama A. 1998 Plasma Phys. Control. Fusion $\mathbf{4 0} 879$

[23] Loarte A. et al 2003 Plasma Phys. Control. Fusion 451549

[24] Perez C.P. et al 2004 Nucl. Fusion 44609 